\title{
Size mismatch: A crucial factor for generating a spin-glass insulator in manganites
}

\author{
A. Maignan and C. Martin \\ Laboratoire CRISMAT, UMR 6508 associée au CNRS, ISMRA et Université de Caen 6, Boulevard du Maréchal Juin, \\ 14050 Caen Cedex, France \\ G. Van Tendeloo \\ EMAT, University of Antwerp (RUCA) Groenenborgerlaan, 171, B 2020, Antwerp, Belgium \\ and Laboratoire CRISMAT, UMR 6508 associée au CNRS, ISMRA et Université de Caen 6, Boulevard du Maréchal Juin, \\ 14050 Caen Cedex, France \\ M. Hervieu and B. Raveau \\ Laboratoire CRISMAT, UMR 6508 associée au CNRS, ISMRA et Universite de Caen 6, Boulevard du Marechal Juin, \\ 14050 Caen Cedex, France \\ (Received 3 March 1999)
}

\begin{abstract}
The structural, electronic, and magnetic properties of the highly mismatched perovskite oxides, $\mathrm{Th}_{0.35} A_{0.65} \mathrm{MnO}_{3}$, where $A$ is for the alkaline earth divalent cations ( $\mathrm{Ca}, \mathrm{Ba}, \mathrm{Sr}$ ), which are all characterized by the same large tolerance factor $(t=0.934)$, have been investigated by using electron microscopy, electrical resistivity, magnetic susceptibility, and magnetization. It is clearly established that a transition from ferromagnetic metallic towards spin-glass insulator samples is induced as the $A$-site cationic size mismatch is increased. Moreover, the magnetoresistance (MR) properties of these manganites are strongly reduced for the spin-glass insulators, demonstrating that the $A$-site cationic disorder is detrimental for the colossal MR properties. Based on these results, a new electronic and magnetic diagram is established that shows that the $A$-site disorder, rather than the $A$-site average cationic size (or $t$ ) is the relevant factor for generating spin-glass insulating manganites.
\end{abstract} [S0163-1829(99)01746-4]

\section{INTRODUCTION}

The numerous investigations of colossal magnetoresistance $(\mathrm{CMR})$ in manganites showed, beside this property, the existence of spin-glass insulator (SGI) behavior for particular compositions. One of the first examples of SGI was discovered in the $\left(\mathrm{La}_{1-x} \mathrm{~Tb}_{x}\right)_{2 / 3} \mathrm{Ca}_{1 / 3} \mathrm{MnO}_{3}$ manganites by Ibarra et al. ${ }^{1-3}$ for $x$ ranging from 0.25 to 0.85 . Then, a similar phenomenon was observed for $\mathrm{Eu}_{0.58} \mathrm{Sr}_{0.42} \mathrm{MnO}_{3}{ }^{4}$ and for $(\mathrm{La}, \mathrm{Y})_{1-x} \mathrm{Ca}_{x} \mathrm{MnO}_{3}$ manganites, ${ }^{5}$ with $x$ ranging from 0.25 to 0.50 . In a recent study of the manganites (La,Dy) ${ }_{0.7} \mathrm{Ca}_{0.3} \mathrm{MnO}_{3},{ }^{6}$ SGI was also observed for tolerance factors smaller than 0.907 .

It is now well established that for a given hole concentration, the magnetic phase diagrams of the manganites are, above all, determined by two factors, the average size of the $A$-site cation of these perovskite oxides, $\left\langle r_{A}\right\rangle$, and the size mismatch at the $A$-site represented by the variance $\sigma^{2}$. The first factor, represented by $\left\langle r_{A}\right\rangle$ or sometimes by the tolerance factor $t$ of the perovskite, influences the Mn-O-Mn bond angles, and thus modifies the transfer integral $(b)$ of $e_{g}$ electron hopping from $\mathrm{Mn}^{3+}$ to $\mathrm{Mn}^{4+}$. Taking into consideration the double-exchange mechanism, ${ }^{7,8}$ responsible for the appearance of the ferromagnetic metallic state (FMM) in these systems, it clearly appears that $b$ increases as $\left\langle r_{A}\right\rangle$ increases, broadening the bandwidth $(\mathrm{W})$, and consequently the Curie temperature $\left(T_{C}\right)$ increases, as shown, for instance, for $\mathrm{Ln}_{0.7} A_{0.3} \mathrm{MnO}_{3}$ manganites. ${ }^{9-13}$ The variance, introduced by Rodriguez-Martinez and Attfield, ${ }^{14}$ defined as $\sigma^{2}$ $=\Sigma y_{1} r_{i}^{2}-\left\langle r_{i}\right\rangle^{2}$, where $y_{i}$ and $r_{i}$ are the fractional occupancies and ionic radii of the $i$ cations, also strongly affects $T_{C}$. As $\sigma^{2}$ increases, local distortions are induced that reduce $T_{C}$ significantly ${ }^{14,15}$ for a constant $\left\langle r_{A}\right\rangle$ value.

The role of these two factors for the appearance of the SGI state is to date not well defined, in contrast to the FMM state. On the basis of the results obtained by the different authors, it was observed that SGI appears below a certain $\left\langle r_{A}\right\rangle$ (or $t$ ) value. Unfortunately, the previous data do not allow us to separate the role of the two parameters, $\left\langle r_{A}\right\rangle$ and $\sigma^{2}$. Our belief is that, size mismatch, since it introduces local distortion and consequently a great variation of the $\mathrm{Mn}$ O-Mn angles, should be a prominent factor for the appearance of spin-glass behavior, rather than the small average size of the $A$ cations. Such an assumption suggests that SGI should be obtained for larger $\left\langle r_{A}\right\rangle$ values, even if the latter tend to favor the FMM state, provided large $\sigma^{2}$ values be involved. Taking into consideration the manganites $\mathrm{Ln}_{0.7} \mathrm{~A}_{0.3} \mathrm{MnO}_{3}$ for which SGI were obtained, ${ }^{1-3,6}$ we have investigated the possibility to synthesize spin-glass insulating manganites for larger $\left\langle r_{A}\right\rangle$ values, increasing the mismatch of the $A$-site cations. We present herein, the study of the highly mismatched series $\mathrm{Th}_{0.35} A_{0.65} \mathrm{MnO}_{3}$, where $A$ represents alkaline earth cations $(\mathrm{Ba}, \mathrm{Ca}, \mathrm{Sr})$. In these manganites, which have the same hole concentration as the $\mathrm{Ln}_{0.7} A_{0.3} \mathrm{MnO}_{3}$ oxides, the $\left\langle r_{A}\right\rangle$ value is maintained constant at a high value of $0.1255 \mathrm{~nm}$ (i.e., $\left\langle r_{A}\right\rangle$ of $\mathrm{Nd}_{0.7} \mathrm{Ba}_{0.3} \mathrm{MnO}_{3}$ ) by varying the nature and ratio of the $A$ cations. $\sigma^{2}$ varies in a large range and can reach high values due to the small size of $\mathrm{Th}^{4+}$ compared to divalent $A$ cations $\left(\mathrm{Ba}^{2+}, \mathrm{Sr}^{2+}, \mathrm{Ca}^{2+}\right)$. From the $\left(T_{C}\right.$ or $\left.T_{g}, \sigma^{2}\right)$ phase diagram, we demonstrate that in spite of the large cationic $A$ site, a SGI state replaces the FMM state when the $A$-site size mismatch is sufficiently high, i.e., $\sigma^{2} \geqslant 2.210^{-4} \mathrm{~nm}^{2}$. 


\section{EXPERIMENT}

For the present investigation nine samples have been prepared by mixing the adequate amounts of $\mathrm{ThO}_{2}, \mathrm{MnO}_{2}$, $\mathrm{CaO}, \quad \mathrm{SrCO}_{3}$, and $\mathrm{BaCO}_{3}$ to satisfy the ratio $\mathrm{Mn}^{3+} / \mathrm{Mn}^{4+}=0.7$ and the average cationic $A$ site $\left\langle r_{A}\right\rangle$ $=0.1255 \mathrm{~nm}$. The powders were heated in air for $12 \mathrm{~h}$ at $1000{ }^{\circ} \mathrm{C}$. The calcined mixtures were reground, pressed in the form of bars, which were sintered in air at $1200^{\circ} \mathrm{C}$ and then at $1500^{\circ} \mathrm{C}$ for $12 \mathrm{~h}$ with a slow cooling until $800^{\circ} \mathrm{C}$.

$\mathrm{X}$-ray diffraction powder data, collected in the $2 \theta$ range of $10-90^{\circ}$ with a step size from $0.02^{\circ}$, confirm the monophasic character of the perovskite structure for each sample. The cell parameters, refined from these records, lead to a nearly constant cell volume in agreement with the constant $\left\langle r_{A}\right\rangle$ value. The cationic compositions determined by energy dispersive spectroscopy are close to the nominal ones. Iodometric titrations evidence an oxygen content of $\mathrm{O}_{3}$, whatever the cationic composition. The electron diffraction (ED) investigation was carried out at room temperature with a JEOL 200 CX electron microscope, the reconstruction of the reciprocal space being made by tilting around the crystallographic axes. High resolution electron microscopy study (HREM) was carried out with a TOPCON 002B electron microscope (200 $\left.\mathrm{kV}, \mathrm{C}_{\mathrm{s}}=0.4 \mathrm{~mm}\right)$.

Resistivity $(\rho)$ was obtained from the standard four-probe method on bars of typical dimensions $10 \mathrm{~mm} \times 1.5$ $\mathrm{mm} \times 1 \mathrm{~mm}$. The indium contacts were ultrasonically deposited on one face of the bar and then connected to the sample holder via copper wires. The temperature $(T)$ or magnetic field $(H)$ dependent resistivity $(\rho)$ measurements were collected with a quantum design physical properties measurements system allowing $T$ and $H$ variations in between 5-400 $\mathrm{K}$ and $0-7 \mathrm{~T}$, respectively. Magnetization measurements were performed with a magnetometer from quantum design within a $5 \mathrm{~T}$ maximum magnetic field value. Alternative current (ac) susceptibility $(\chi)$ as a function of $T$ was collected with a Lake-Shore 7000 susceptometer and an ac/dc superconducting quantum interference device magnetometer by using ac magnetic fields in the range 3-10 Oe and frequencies varying between 1 and $1 \mathrm{kHz}$ without applying a dc magnetic field.

\section{RESULTS}

The samples from this $\mathrm{Th}_{0.35} \mathrm{~A}_{0.65} \mathrm{MnO}_{3}$ series differ only by their $A$-site size mismatch and consequently will be labeled in the present paper by their $\sigma^{2}$ value, which varies from $1.74 \times 10^{-4} \mathrm{~nm}^{2}$ to $2.80 \times 10^{-4} \mathrm{~nm}^{2}$ (Table I). The high crystalline quality of these pure samples is only shown for the more mismatched one. The [010] and [111] ED patterns of Figs. 1(a) and 1(b), respectively, evidence an I-type lattice. The sharp spots indicate the good crystallinity of the samples, while the absence of extra reflections and/or diffuse streaks suggests the absence of long- or short-range ordering phenomena. This was confirmed by the HREM study. Along all viewing directions, the HREM images exhibit a very regular contrast [Fig. 1(c)], characteristic of the perovskiterelated structures.

The $\rho(T)$ curves of these manganites [Fig. 2(a)], registered on cooling in the absence of magnetic field, demon-
TABLE I. $\sigma^{2}$ and $A$ contents $(x, y, z)$ for the $\mathrm{Th}_{0.35}\left(\mathrm{Ba}_{x} \mathrm{Sr}_{y} \mathrm{Ca}_{z}\right) \mathrm{MnO}_{3}$ series.

\begin{tabular}{lllc}
\hline \hline$x(\mathrm{Ba})$ & $y(\mathrm{Sr})$ & $z(\mathrm{Ca})$ & $\sigma^{2} \times 10^{4}\left(\mathrm{~nm}^{2}\right)$ \\
\hline 0.138 & 0.512 & 0 & 1.74 \\
0.16 & 0.463 & 0.027 & 1.84 \\
0.18 & 0.419 & 0.051 & 1.96 \\
0.20 & 0.374 & 0.076 & 2.00 \\
0.22 & 0.329 & 0.101 & 2.12 \\
0.24 & 0.285 & 0.125 & 2.20 \\
0.26 & 0.24 & 0.15 & 2.30 \\
0.30 & 0.15 & 0.20 & 2.47 \\
0.3675 & 0 & 0.2825 & 2.80 \\
\hline \hline
\end{tabular}

strate that the insulator to metal (IM) transition can be suppressed by increasing $\sigma^{2}$ beyond a certain value $\left(\sigma^{2}\right.$ $>2.12 \times 10^{-4} \mathrm{~nm}^{2}$ ), although $\left\langle r_{A}\right\rangle$ is high and remains constant for all the series $(0.1255 \mathrm{~nm})$. Correlatively for lower $\sigma^{2}$ values $\left(\sigma^{2} \leqslant 2.12 \times 10^{-4} \mathrm{~nm}^{2}\right)$, the transition temperature $T_{\text {IM }}$ decreases rapidly as $\sigma^{2}$ increases, from $150 \mathrm{~K}$ for $\sigma^{2}$ $=1.84 \times 10^{-4} \mathrm{~nm}^{2}$ to $50 \mathrm{~K}$ for $\sigma^{2}=2.12 \times 10^{-4} \mathrm{~nm}^{2}$, whereas the resistivity at $5 \mathrm{~K}$ increases dramatically from $3 \times 10^{-2} \Omega \mathrm{cm}$ to $3 \times 10^{4} \Omega \mathrm{cm}$, respectively. The combined decrease of $T_{\mathrm{IM}}$ and increase of $\rho_{5 \mathrm{~K}}$ can easily be explained by a weakening of the ferromagnetic metallic (FMM) state as $\sigma^{2}$ increases, which finally disappears for $\sigma^{2}$ $>2.12 \times 10^{-4} \mathrm{~nm}^{2}$. This statement, based on the doubleexchange scenario, is strongly supported by the ac susceptibility curves $\chi^{\prime}(T)$ [Fig. 2(b)]. One indeed observes that for low mismatch values, i.e., $\sigma^{2}<2.00 \times 10^{-4} \mathrm{~nm}^{2}$, the curves are characteristic of a good ferromagnet, so that the classical FMM state is obtained, whereas for larger mismatch, i.e., $\sigma^{2} \geqslant 2.00 \times 10^{-4} \mathrm{~nm}^{2}$, curves with one or two maxima, in-

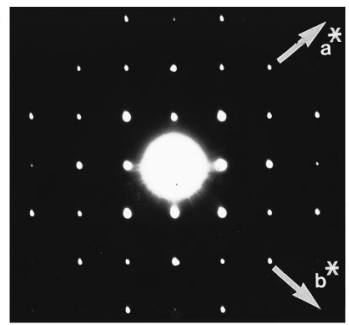

(a)

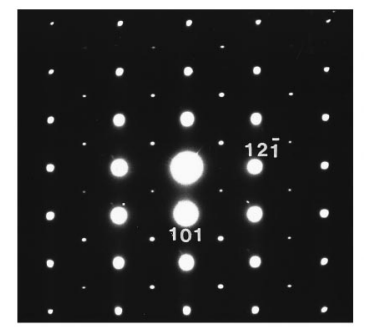

(b)

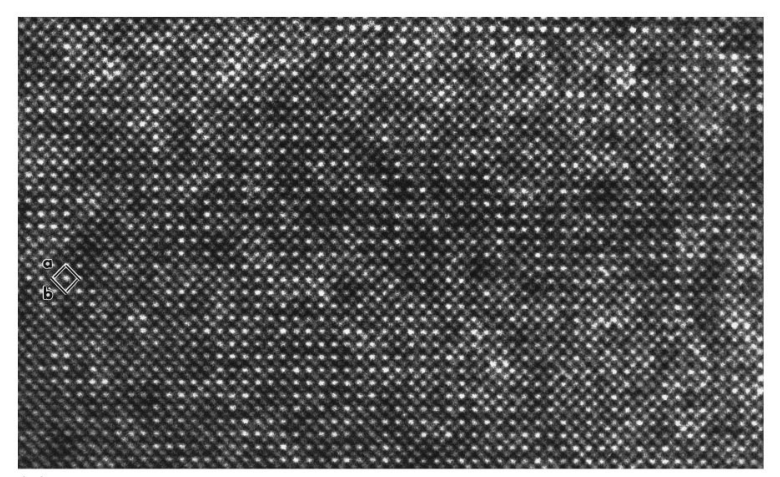

(c)

FIG. 1. [010] (a) and [111] (b) electron diffraction patterns of $\mathrm{Th}_{0.35} \mathrm{Ba}_{0.3675} \mathrm{Ca}_{0.2825} \mathrm{MnO}_{3}\left(\sigma^{2}=2.80 \times 10^{-4} \mathrm{~nm}^{2}\right)$. Corresponding high resolution image $(\mathrm{c})$. 

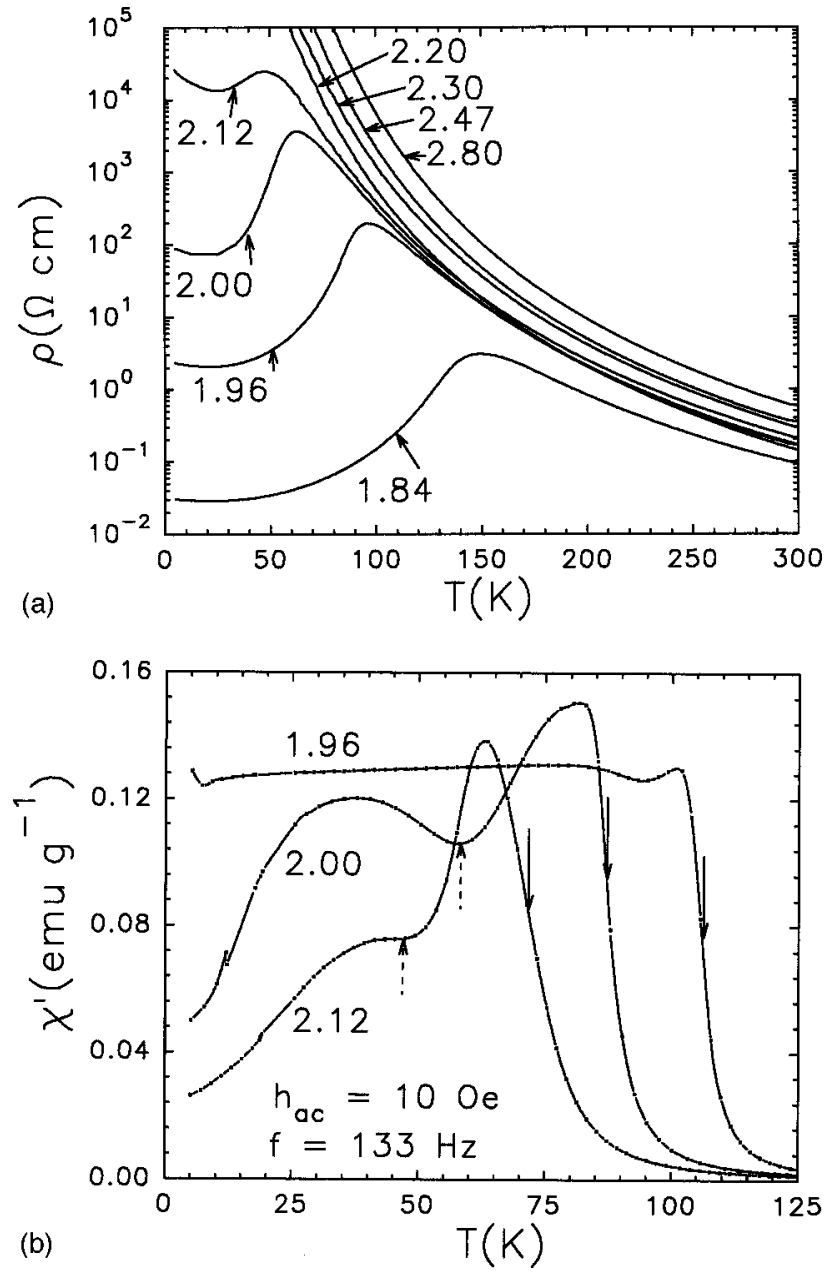

FIG. 2. (a) $T$-dependent resistivity ( $\rho$ ) of the $\mathrm{Th}_{0.35} A_{0.65} \mathrm{MnO}_{3}$ samples registered in the absence of magnetic field. $\sigma^{2} \times 10^{4}\left(\mathrm{~nm}^{2}\right)$ values are labeled on the graph. (b) $T$-dependent real part of the ac susceptibility ( $\chi^{\prime}$ ) for the $\mathrm{Th}_{0.35} A_{0.65} \mathrm{MnO}_{3}$ samples (10 Oe and $f$ $=133 \mathrm{~Hz}$ ). The solid vertical arrows indicate the inflection points determining $T_{C}$. Dashed vertical arrows indicate the $\chi^{\prime}$ minimum temperature, which correspond to the $\rho$ maximum on (a).

dicative of unconventional ferromagnetism, are obtained. Clearly, the existence of double peaks on the $\chi^{\prime}(T)$ curves for samples with $\sigma^{2}=2.00 \times 10^{-4} \mathrm{~nm}^{2}$ and $2.12 \times 10^{-4} \mathrm{~nm}^{2}$ strongly suggests the presence of ferromagnetic finite-size clusters for these compositions, as already probed for highly $A$-site mismatched $(\mathrm{La}, \mathrm{Tb})_{0.7} \mathrm{Ca}_{0.3} \mathrm{MnO}_{3}$ manganites by small angle neutron scattering (SANS). ${ }^{1}$ Note that for our two samples, $T_{\mathrm{IM}}$ deduced from the $\rho(T)$ curves does not coincide with $T_{C}$ deduced from the derivative maximum on the $\chi^{\prime}(T)$ curves [see vertical solid arrows on Fig. 2(b)], but rather with the minimum located between the two maxima (dashed arrows). Thus for these compositions, long-range ferromagnetism disappears and consequently hinders metallicity, leading to high resistivity at $5 \mathrm{~K}$. The existence of local atomic disorder introduced by the $A$-cations size mismatch, affecting the FMM state installation, also strongly modifies the resistivity in the paramagnetic metallic insulating (PMI) region. A clear increase of $\rho_{300 \mathrm{~K}}$ with $\sigma^{2}$ is observed on Fig. 2(a), emphasizing the effect of local disorder on the carriers scattering.

For higher mismatch, $\sigma^{2}>2.12 \times 10^{-4} \mathrm{~nm}^{2}$, the $\chi^{\prime}(T)$

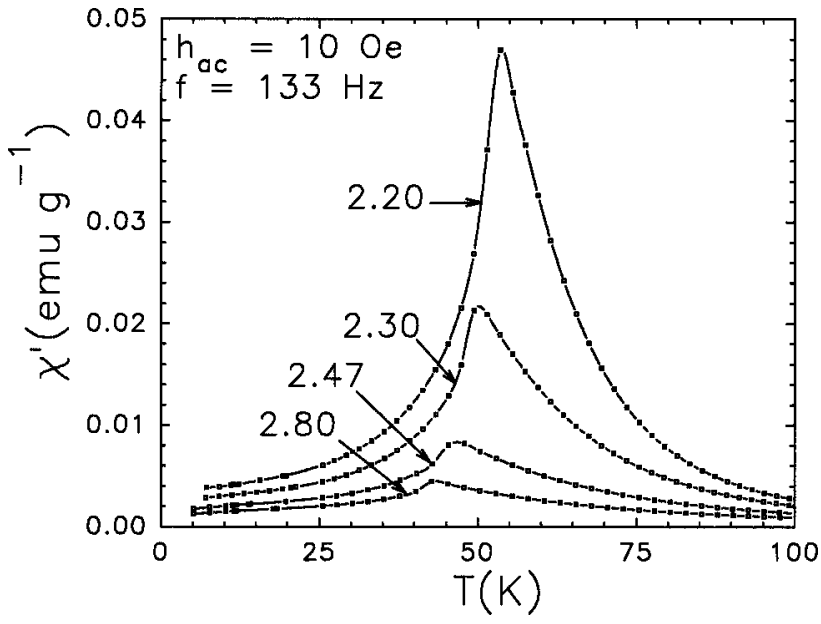

FIG. 3. $\chi^{\prime}(T)$ curves of samples with the higher mismatched values.

curves show a cusp (Fig. 3), suggesting a spin-glass insulator (SGI) behavior, owing to the great similarity with the curves reported for the SGI (La, Tb) ${ }_{0.7} \mathrm{Ca}_{0.3} \mathrm{MnO}_{3}$ (Refs. 1-3) and $(\mathrm{La}, \mathrm{Y})_{0.5} \mathrm{Ca}_{0.5} \mathrm{MnO}_{3}$ (Ref. 5) on the basis of SANS data, magnetization and aging experiments. The temperature $T_{g}(g$ is for "glass") of the $\chi^{\prime}$ maximum decreases as $\sigma^{2}$ increases. More importantly, it must be emphasized that as soon as a cusp is obtained $\left(\sigma^{2} \geqslant 2.20 \times 10^{-4} \mathrm{~nm}^{2}\right)$ the IM transition is suppressed on the corresponding $\rho(T)$ curves [Fig. 2(a)]. The remaining ferromagnetic interactions, if any, are at sufficiently short range to hinder any kind of charge delocalization in the absence of magnetic field. This is corroborated by the isothermal field dependent magnetization $(M)$ measurements collected at $10 \mathrm{~K}$ (Fig. 4). For compositions with an IM transition and an ac- $\chi$ curve characteristic of a ferromagnet (illustrated for $\sigma^{2}=1.96 \times 10^{-4} \mathrm{~nm}^{2}$ in Fig. 4 ), a saturated magnetization above $\sim 1 \mathrm{~T}$, corresponding to $3.7 \mu_{B}$ per manganese, as expected from the formula, is obtained together with a very small hysteresis in the zero field vicinity, as in soft ferromagnets. On the opposite, samples characterized by ac- $\chi$ curve with a cusp do not show any saturation even in $5 \mathrm{~T}\left(\sigma^{2}=2.20 \times 10^{-4} \mathrm{~nm}^{2}\right.$ in Fig. 4), and

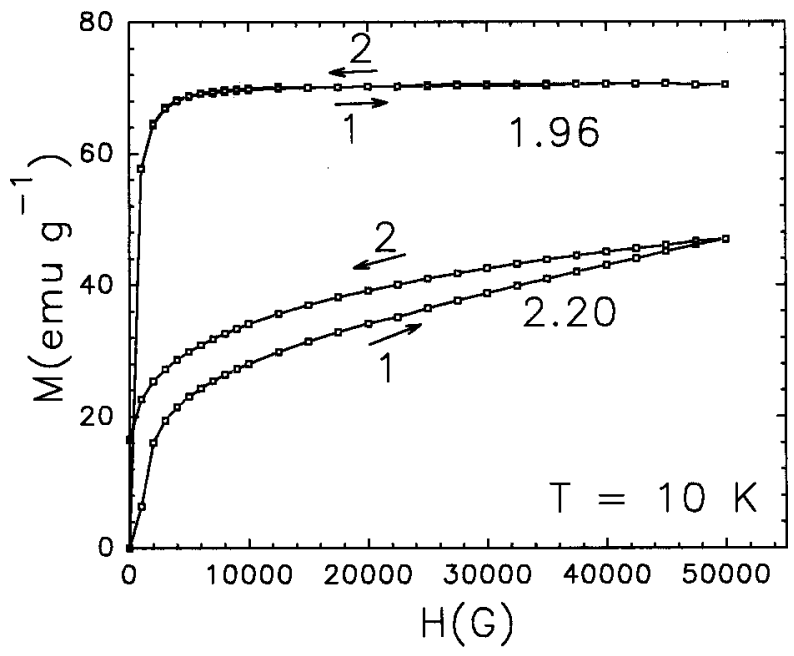

FIG. 4. $H$-dependent magnetization $(M)$ curves registered at 10 K. 1 and 2 are for the increasing and decreasing branches, respectively. 


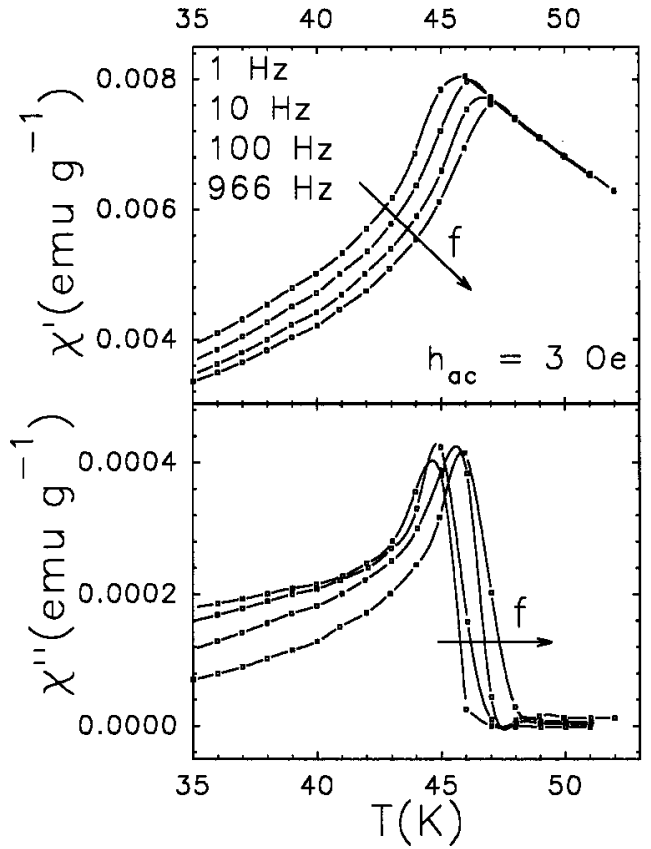

FIG. 5. Real part $\chi^{\prime}(T)$ (top panel) and imaginary part $\chi^{\prime \prime}(T)$ (low panel) of the ac susceptibility for the sample with $\sigma^{2}$ $=2.47 \times 10^{-4} \mathrm{~nm}^{2}$ registered with the four frequencies labeled on the graph $\left(h_{\mathrm{ac}}=3 \mathrm{Oe}\right)$. The arrows indicate the increasing frequencies.

the $M(H)$ curves are strongly hysteretic. Moreover, the first magnetization curve exhibits an $S$ shape, which is characteristic of the spin-glass-like behavior.

In order to confirm the spin-glass state, whose cusp on the $\chi^{\prime}(T)$ curve is related to relaxation phenomena, $\chi^{\prime}(T)$ and $\chi^{\prime \prime}(T)$ curves have been registered for four frequencies covering three decades, for a highly mismatched compound with $\sigma^{2}=2.47 \times 10^{-4} \mathrm{~nm}^{2}$ (Fig. 5). The $T_{g}$ increase with $f$ is characteristic of the spin-glass state and is usually estimated from the quantity $K,{ }^{16} K=\Delta T_{g} / T_{g} \Delta\left(\log _{10} f\right)$, where $\Delta$ refers to difference. By taking $T_{g}$ on the $\chi^{\prime}(T)$ curves or from the inflection points on right part of the $\chi^{\prime \prime}(T)$ curves of Fig. 5, one obtains $K=1.1 \times 10^{-2}$, which is an expected value for a typical spin glass, if one refers to the values $K=5.0$ $\times 10^{-3}, 1.8 \times 10^{-2}$, and $1.0 \times 10^{-2}$ for CuMn, NiMn, and $\mathrm{Ho}_{2} \mathrm{Mn}_{2} \mathrm{O}_{7}$, respectively. ${ }^{16,17}$ The spin-glass state of this thorium-based manganite is also confirmed from dc magnetization measurements, registered on warming in $10 \mathrm{Oe}$ and 100 Oe upon field cooling (FC) and after a zero-field cooling (ZFC) process (Fig. 6), which clearly show a branching at $T_{g}$ of the FC and ZFC data, FC branches being rather flat below $T_{g}$ as usually observed for a SG. ${ }^{16}$

These magnetic and transport measurements clearly establish that a FMM-to-SGI transition appears as $\sigma^{2}$ increases, $\left\langle r_{A}\right\rangle$ and hole concentration being constant. The relationships between the CMR properties, magnetic states, and $\sigma^{2}$ have also been studied. A clear correlation with the FMM/ SGI transition is evidenced. As soon as a cusp appears on the ac- $\chi^{\prime}$ curves, characterizing the SGI state, a magnetic field of $7 \mathrm{~T}$ is no more sufficient to restore a FMM state as shown from Fig. 7(a) for the sample with $\sigma^{2}=2.30 \times 10^{-4} \mathrm{~nm}^{2}$. In contrast, even for compounds exhibiting an incomplete IM transition in the absence of magnetic field, a large magne-

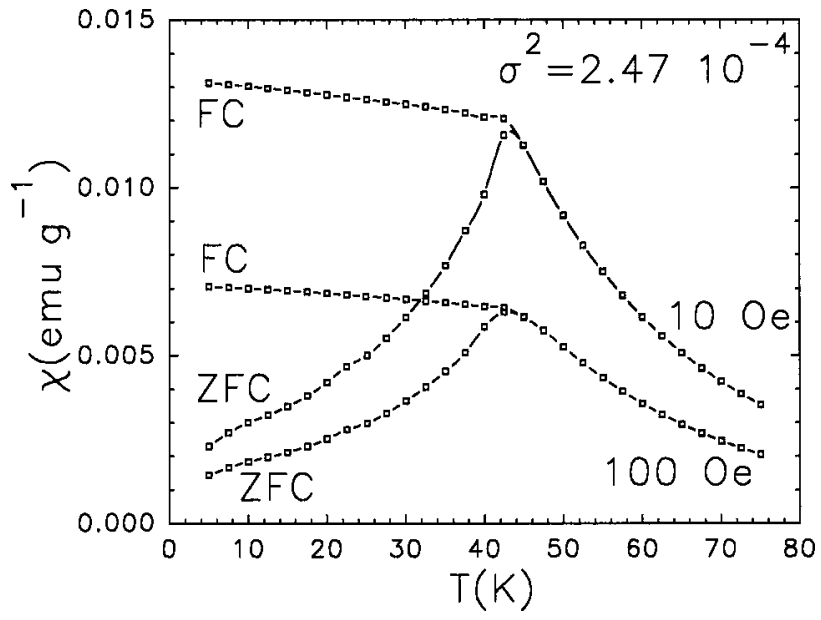

FIG. 6. $\chi(T)$ curves obtained from $M(T)$ data collected in 10 and 100 Oe in-field cooled mode (FC) and after a zero-field cooling process (ZFC) for the $\sigma^{2}=2.47 \times 10^{-4} \mathrm{~nm}^{2}$ sample.

toresistance effect is obtained as shown from the comparison of $\rho(T)$ curves registered on cooling in 0 and $7 \mathrm{~T}$ for $\sigma^{2}$ $=2.12 \times 10^{-4} \mathrm{~nm}^{2}$ [Fig. 7(b)].

It must also be pointed out that in the mismatched manganites, there exists a low field magnetoresistance as reported for $\mathrm{Nd}_{0.7} \mathrm{Ba}_{0.3} \mathrm{MnO}_{3}$ (Ref. 18) (same $\left\langle r_{A}\right\rangle$ value, $0.1255 \mathrm{~nm}$, and $\sigma^{2}=1.98 \times 10^{-4} \mathrm{~nm}^{2}$ ) studied in both ceramic and single-crystal forms and which has been ascribed to the presence of strains in grain boundaries. This kind of behavior is also encountered in these Th-based polycrystalline samples [Fig. 7(c) for the $\sigma^{2}=1.74 \times 10^{-4} \mathrm{~nm}^{2}$ sample], the isothermal $\rho(H)$ curve showing first an abrupt decrease of resistivity below $0.1 \mathrm{~T}$ and a less rapid regime above. Thus, the first regime at low field originates at the interfaces between grains and it has been interpreted as tunneling magnetoresistance. ${ }^{19}$

\section{DISCUSSION}

The present investigation of highly mismatched manganites, with rather a high $\left\langle r_{A}\right\rangle$ value (or high tolerance factor $t$ ), shows that the $\sigma^{2}$ variance is the crucial parameter that governs the appearance of the SGI state. In other words, for this hole concentration, previously studied for the oxides (La,Dy) ${ }_{0.7} \mathrm{Ca}_{0.3} \mathrm{MnO}_{3},{ }^{6} \quad(\mathrm{La}, \mathrm{Pr})_{0.7}\left(\mathrm{Ca}_{0.3}\right) \mathrm{MnO}_{3},{ }^{9}$ $(\mathrm{La}, \mathrm{Tb})_{0.7} \mathrm{Ca}_{0.3} \mathrm{MnO}_{3},{ }^{1-3}$ and $(\mathrm{La}, \mathrm{Y}){ }_{1-x} \mathrm{Ca}_{x} \mathrm{MnO}_{3},{ }^{5}$ the tolerance factor $t$, or the $\left\langle r_{A}\right\rangle$ value, cannot be considered as a limiting parameter for the appearance of spin glass. Thus the critical value of $t=0.907$, above which SGI does not appear, ${ }^{6}$ cannot be retained as a criterion for all the manganites with the same hole concentration, since SGI is obtained herein for $t=0.934$. The difficulty of interpretation of the previous results ${ }^{1-3,6}$ comes from the fact that in these manganites, which have the same hole concentration, both $\left\langle r_{A}\right\rangle$ (or $t$ ) and $\sigma^{2}$ vary simultaneously, so that the relative influence of the two factors could not be established.

From the present observations, it appears clearly that phase diagrams ( $T_{g}$ or $T_{C}$ vs $\sigma^{2}$ ) have to be established for each series of manganites keeping $\left\langle r_{A}\right\rangle$ and hole concentration constant. Such a diagram is shown in Fig. 8 for $\mathrm{Th}_{0.35} A_{0.65} \mathrm{MnO}_{3}$ manganites with $\left\langle r_{A}\right\rangle=0.1255 \mathrm{~nm}$ (or $t$ 

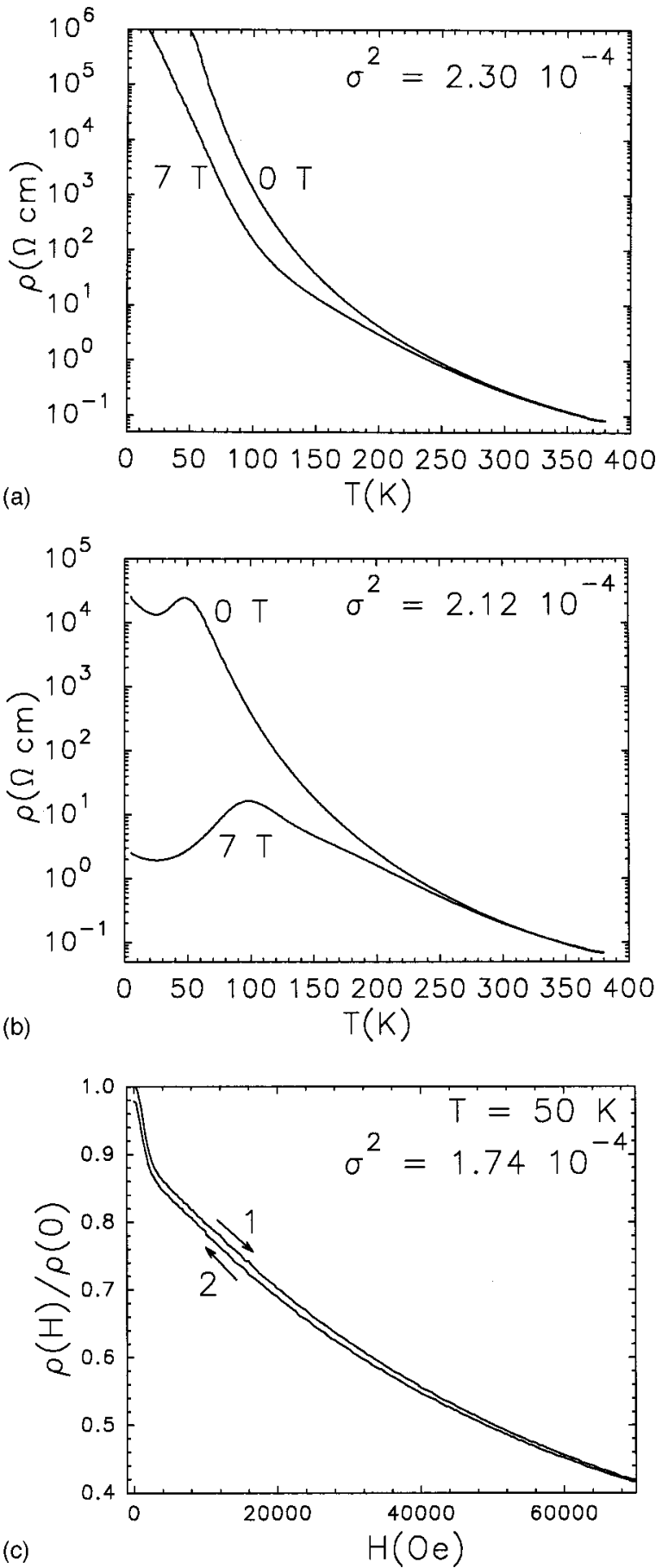

FIG. 7. $\rho(T)$ curves registered upon cooling in zero field (0) and in $7 \mathrm{~T}$ for the samples characterized by $\sigma^{2}=2.30 \times 10^{-4} \mathrm{~nm}^{2}$ (a) and $\sigma^{2}=2.12 \times 10^{-4} \mathrm{~nm}^{2}$ (b). (c) $\rho(H) / \rho(0)$ curve registered at 50 $\mathrm{K}$ for the compound of $\sigma^{2}=1.74 \times 10^{-4} \mathrm{~nm}^{2}$ ( 1 and 2 are for the ascending and decreasing branches, respectively).

$=0.934)$. The $x$ axis has been intentionally inverted to obtain a diagram that mimics the $\left(T_{g}\right.$ or $T_{C}$ vs $\left.t\right)$ one reported previously for $(\mathrm{La}, \mathrm{Tb})_{0.7} \mathrm{Ca}_{0.3} \mathrm{MnO}_{3}$ and (La,Dy) ${ }_{0.7} \mathrm{Ca}_{0.3} \mathrm{MnO}_{3}$ systems. ${ }^{1-3,6}$ Similarly, three different regions-PMI, FMM, and SGI-can be distinguished. Note the spectacular decrease of $T_{C}$ from $150 \mathrm{~K}$ to $70 \mathrm{~K}$, corresponding to the FMM-PMI transition, due to the only $\sigma^{2}$ increase, in agreement with the results reported by Rodriguez-Martinez and Attfield. ${ }^{14}$ By increasing $\sigma^{2}$ further,

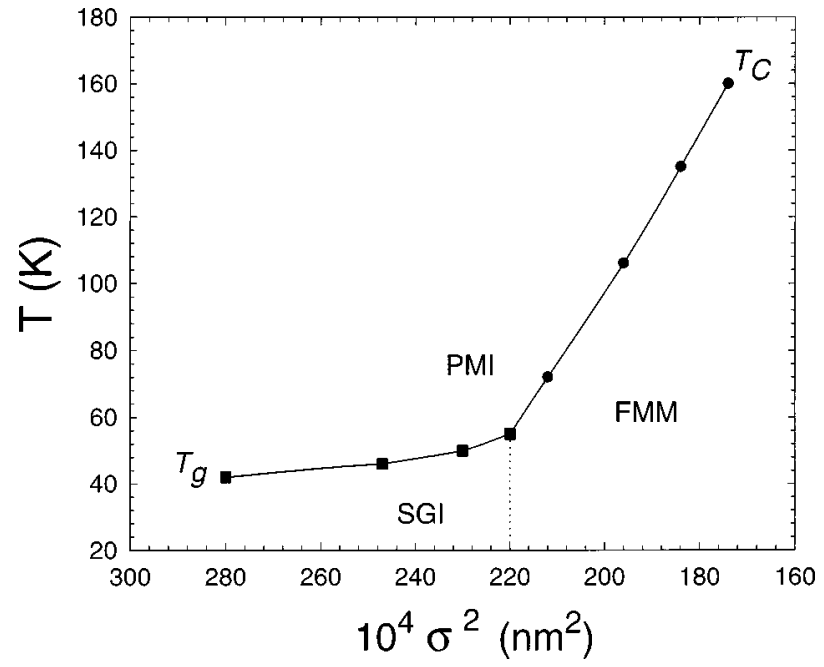

FIG. 8. Electronic and magnetic diagram established for the $\mathrm{Th}_{0.35} A_{0.65} \mathrm{MnO}_{3}$ series: $T_{C}(\bigcirc)$ and $T_{g}(\bigcirc)$ as a function of the variance $\sigma^{2}$. The boundary between the SGI and FMM regions is symbolized by the dotted line.

the FMM state is replaced by the SGI state (for $\sigma^{2}$ $\geqslant 2.20 \times 10^{-4} \mathrm{~nm}^{2}$ ), and $T_{g}$, which separates the SGI and PMI states, decreases only slightly.

Based on these results, it appears most probable that SGI will be defavored for too small $\left\langle r_{A}\right\rangle$ values in the $\mathrm{Ln}_{0.70} A_{0.30} \mathrm{MnO}_{3}$ compounds, due to the fact that when $\left\langle r_{A}\right\rangle$ becomes smaller, we can only get small $\sigma^{2}$ values. This statement is supported by the magnetic properties of the manganite $\mathrm{Dy}_{0.7} \mathrm{Ca}_{0.3} \mathrm{MnO}_{3}$, which does not exhibit a cusp on its $\chi^{\prime}(T)$ curve (Fig. 9) in spite of its $\left\langle r_{A}\right\rangle$ value smaller than that of the SGI $\mathrm{La}_{0.35} \mathrm{Dy}_{0.35} \mathrm{Ca}_{0.3} \mathrm{MnO}_{3}$ (Fig. 9). This result is thus in agreement with the weaker $A$-site cationic size mismatch of the former.

The spin-glass insulating state induced by $A$-site cationic size disordering differs thus from the magnetic behavior reported for the $\mathrm{La}_{0.5} \mathrm{Sr}_{0.5} \mathrm{CoO}_{3}$ cobaltite and the $B$-site substituted $\mathrm{La}_{0.7} \mathrm{Sr}_{0.3} \mathrm{Mn}_{1-x} \mathrm{Co}_{x} \mathrm{O}_{3}$ manganites for which the

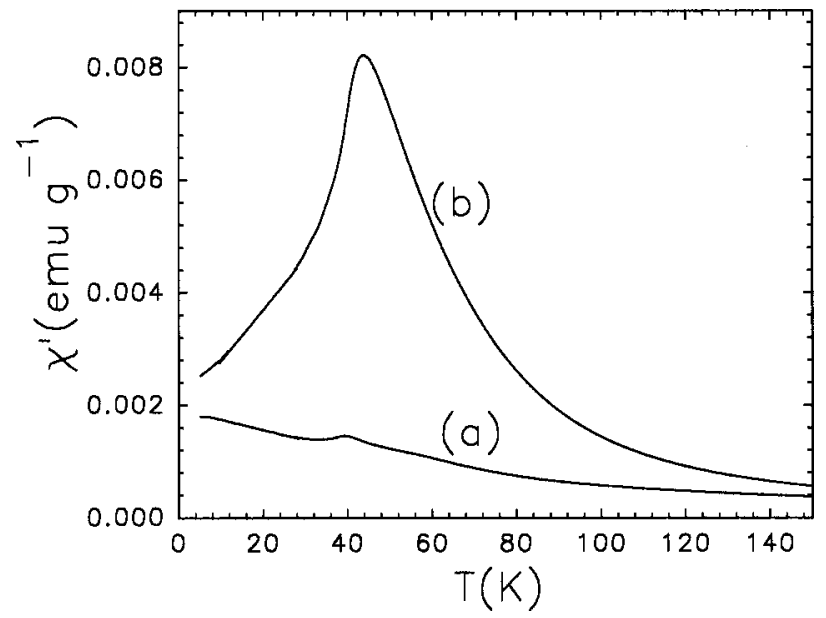

FIG. 9. $\chi^{\prime}(T)$ curves of $\mathrm{Dy}_{0.7} \mathrm{Ca}_{0.3} \mathrm{MnO}_{3}\left[\left\langle r_{A}\right\rangle=0.1120 \mathrm{~nm}\right.$ and $\left.\sigma^{2}=1.97 \times 10^{-5} \mathrm{~nm}^{2}\right] \quad$ (a) and $\mathrm{Dy}_{0.35} \mathrm{La}_{0.35} \mathrm{Ca}_{0.3} \mathrm{MnO}_{3} \quad\left[\left\langle r_{A}\right\rangle\right.$ $=0.1158 \mathrm{~nm}$ and $\left.\sigma^{2}=3.29 \times 10^{-5} \mathrm{~nm}^{2}\right]$ (b). $h_{\mathrm{ac}}=10$ Oe and $f$ $=133 \mathrm{~Hz}$ 
cluster-glass state is believed to result from ferromagnetic dilution. $^{20-22}$ The origin of magnetic frustration in the present SG insulators is more probably connected with the incoherent displacements of the oxygen atoms bonded to the Mn cations, which have been reported to increase with the $A$-site cationic size mismatch. ${ }^{23}$ The random distribution of the local distortions in the $\mathrm{MnO}_{3}$ network facilitates the stabilization of static Jahn-Teller distorted $\mathrm{Mn}^{3+} \mathrm{O}_{6}$ octahedra.
Consequently, the resulting random magnetic anisotropy is probably the responsible mechanism for the frustration.

\section{ACKNOWLEDGMENTS}

The authors gratefully acknowledge the University of CAEN, for supporting this work through a position of associate professor. Gustaaf Van Tendeloo acknowledges the IUAP 4/10 program of the Belgian government.
${ }^{1}$ M. R. Ibarra and J. M. de Teresa, in Colossal Magnetoresistance, Charge Ordering and Related Properties of Manganese Oxides, edited by C. N. R. Rao and B. Raveau (World Scientific, Singapore, 1998), pp. 83-154.

${ }^{2}$ J. M. de Teresa, M. R. Ibarra, J. Blasco, C. Ritter, P. A. Algarabel, C. Marquina, and A. del Moral, Phys. Rev. Lett. 76, 3392 (1996).

${ }^{3}$ J. M. De Teresa, C. Ritter, M. R. Ibarra, P. A. Algarabel, J. L. Garcia-Munoz, J. Blasco, J. Garcia, and C. Marquina, Phys. Rev. B 56, 3317 (1997).

${ }^{4}$ A. Sundaresan, A. Maignan, and B. Raveau, Phys. Rev. B 55, 5596 (1997).

${ }^{5}$ A. Maignan, A. Sundaresan, U. V. Varadaraju, and B. Raveau, J. Magn. Magn. Mater. 83, 184 (1998).

${ }^{6}$ T. Terai, T. Kakeshita, T. Fukuda, T. Saburi, N. Takamoto, K. Kindo, and M. Honda, Phys. Rev. B 58, 14908 (1998).

${ }^{7}$ C. Zener, Phys. Rev. 82, 403 (1951).

${ }^{8}$ P. G. de Gennes, Phys. Rev. 118, 141 (1960).

${ }^{9}$ H. Y. Hwang, S. W. Cheong, P. G. Radaelli, M. Marezio, and B. Batlogg, Phys. Rev. Lett. 75, 914 (1995).

${ }^{10}$ P. G. Radaelli, M. Marezio, H. Y. Hwang, and S. W. Cheong, J. Solid State Chem. 122, 444 (1996).

${ }^{11}$ R. Mahesh, R. Mahendiran, A. K. Raychaudhuri, and C. N. R. Rao, J. Solid State Chem. 120, 204 (1995).
${ }^{12}$ B. Raveau, A. Maignan, and V. Caignaert, J. Solid State Chem. 117, 224 (1995).

${ }^{13}$ A. Maignan, Ch. Simon, V. Caignaert, and B. Raveau, Z. Phys. B 99, 305 (1996).

${ }^{14}$ L. M. Rodriguez-Martinez and J. P. Attfield, Phys. Rev. B 54, 15622 (1996).

${ }^{15}$ F. Damay, C. Martin, A. Maignan, and B. Raveau, J. Appl. Phys. 82, 6181 (1997).

${ }^{16}$ J. A. Mydosh, Spin Glasses (Taylor \& Francis, London, 1993).

${ }^{17}$ J. E. Greedan, N. P. Raju, A. Maignan, Ch. Simon, J. S. Pedersen, A. M. Nirainathi, E. Gmelin, and M. A. Subramanian, Phys. Rev. B 54, 7189 (1996).

${ }^{18}$ A. Maignan, C. Martin, M. Hervieu, B. Raveau, and J. Hejtmanek, Solid State Commun. 107, 363 (1998).

${ }^{19}$ H. Y. Hwang, S. W. Cheong, N. P. Ong, and B. Battlog, Phys. Rev. Lett. 77, 2041 (1996).

${ }^{20}$ D. N. H. Nam, K. Jonason, P. Nordblad, N. V. Khiem, and N. X. Phuc, Phys. Rev. B 59, 4189 (1999).

${ }^{21}$ N. Gayathri, A. K. Raychaudhuri, S. K. Tiwary, R. Gundakaram, A. Arulraj, and C. N. R. Rao, Phys. Rev. B 56, 1345 (1997).

${ }^{22}$ X. G. Li, X. J. Fan, G. Ji, W. B. Wu, K. H. Wong, C. L. Choy, and H. C. Ku, J. Appl. Phys. 85, 1663 (1999).

${ }^{23}$ L. M. Rodriguez-Martinez and J. P. Attfield, Chem. Mater. 11, 1504 (1999). 